\title{
Survivability Issues in Optical Wavalength Division Multiplexing (WDM) Network
}

\author{
Bhas Raj Pathak ${ }^{1}$ and Shrikant S. Tangade ${ }^{2}$ \\ Dept. of Electronics and Communication Engg. REVA Institute of Technology and Management, Bengaluru, \\ India \\ Dept. of Electronics and Communication Engg. REVA Institute of Technology and Management, Bengaluru, \\ India
}

\begin{abstract}
This paper provides a survey on existing survivability related problems and include the new algorithm based on existing algorithms. Survivability has become an important issue and topical subject in WDM networks. This paper reviewed the literature on survivability against multi-domain or link failures to ensure reliability in WDM networks. Survivability in multi-domain optical networks is very important, only few studies have been proposed in literature. The paper is related to protection technique in inter-domain link of multi-domain network combining the p-cycle and wavelength assignment.
\end{abstract}

Keywords-WDM, Survivability, Routing, Lightpath, protection, Optical Networks, p-cycle, multi-domain.

\section{INTRODUCTION}

The development of Wavelength Division Multiplexing (WDM) technologies has increased enormously the transmission capacity of optical networks [2]. Wavelength division multiplexing technology is used in optical networks that allow to support high bandwidth application s such as HDTV, videoconferencing. To meet the thousand-fold growth requirements, it is challenged to build large-scale electronic routers and switches in a cost effective way with reduces power consumption. Other than cost issue, survivability is a critical concern in optical network design since the failure of a network element can cause a huge amount of data loss [10]. Survivability is one of the major issues that are developing in WDM networks [3]. Survivability means that the network has the ability to maintain the acceptable level of service even after a failure within the network. This requirement becomes more critical as the size and the usage of network increases.

Network "survivability" is not the purely an academic subject in the real telecommunication networks, failures happens quite frequently and with catastrophic consequences. Failures in optical networks can be distinguished depending whether they damage links or switching devices. Single domain networks comprises of different nodes connected together either in ring topology or mesh topology. In a single domain network basic problem may be defined as: Given a network topology, a set of candidate working paths, and a forecast of the point-to-point demand traffic, determine the routing for each point-to-point demand and the least cost WDM equipment configuration required to support these routes [6]. A multi-domain networks are composed of several single single-domain networks interconnected by inter-domain links. Each single domain can be regarded as an independent network that has its own local rules of operation and management to provide services. Due to scalability constraints and management policies, the internal topological details of a domain are usually not shared externally. As a result, no node in a multi-domain network can have complete information in multidomain network. The protection in multi-domain network is difficult than that of single domain network.

The rest of the paper is organized as follows. In section II we present WDM networks and basic concepts about WDM networks. In section III, we describe the features of multi-domain networks. In section IV, we discussing about general concepts and the elements related to survivability in multi-domain network and discussing their capabilities and performance. In section $\mathrm{V}$ we discuss new algorithm as per the proposed work. Finally we concluded in section VI.

\section{WDM NETWORKS: BASIC CONCEPTS}

Modern optical networks are complex systems designed according to a layered approach. Higher layer are fully managed by electronic equipments. Several protocols can be stacked one over other in various combination (IP over SDH, IP over ATM, ATM over the Ethernet, ATM over SDH and so on). The main task of WDM layer is connectivity and bandwidth provisioning to the electronic layers in client server relationship [4]. The provisioning service offered by WDM consist of setting up optical point to point circuits in order to fulfill request of a point to point connection request issued by the upper layer. Such a request defines a so called virtual connection while optical circuit is named lightpath. A lightpath is set up by reserving to the virtual connection a sequence of WDM channels linking the source to the destination node throughout the optical network. 
The WDM protocol layer is the set of protocol created to control and manage the so called optical transport network. According to ITU-T Recommendation G.872, WDM layer is divided into four sub-layers: the optical channel sublayer, the optical multiplex section sublayer(OMS), the optical transmission section sublayer(OTS) and physical media sublayer.

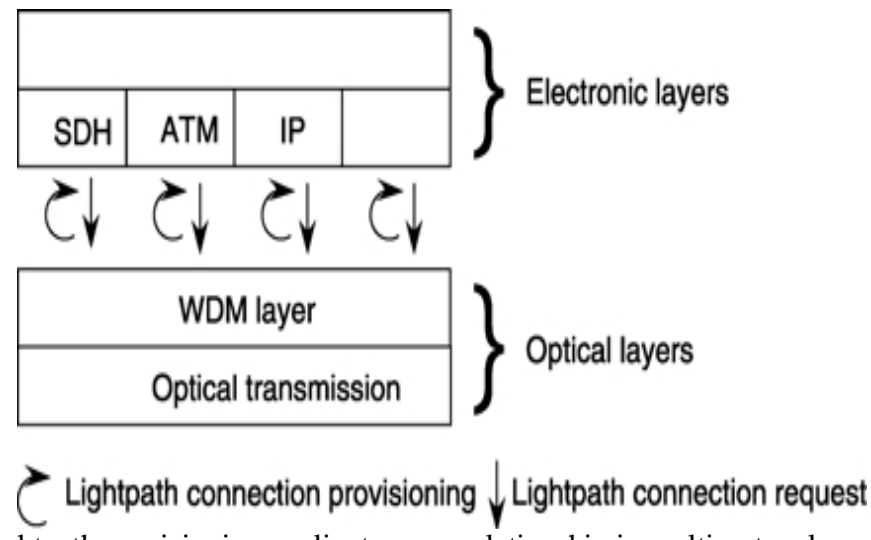

Fig. 1 Lightpath provisioning: a client server relationship in multiprotocol environment.

\begin{tabular}{|l|c|}
\hline \multicolumn{2}{|c|}{ Electronic Layers } \\
\hline \multirow{3}{*}{$\sum_{3}$} & OCh - Optical Channel \\
\cline { 2 - 2 } & OMS - Optical Multiplex Section \\
\cline { 2 - 2 } & OTS - Optical transmission Section \\
\cline { 2 - 2 } & Physical media (optical fiber) \\
\hline
\end{tabular}

Fig. 2 Optical-Transport-Network protocol architecture: sublayers of the WDM layer.

The main entity managed by the optical channel sublayer is the lightpath. OCh takes care of all the end to end networking functions: routing and wavelength assignment, connectivity check, fault recovery. OCh signaling is carried by an optical supervisory channel (OCh-OSc): several techniques to associate the OCh-OCs information to each lightpath have been proposed.

The optical multiplex section sublayer control not only single path but the multiplex of all the WDM channels that transmits on a single fiber, each one carried by a particular wavelength. OMs mainly performs WDM multiplex monitoring. Signaling for OMs is provided by an optical supervising channel, normally transmitted by using a dedicated wavelength. OTs absolves all the control operation required to manage and supervise the optical transmission devices (amplifiers, transponders, regenerators and so on). OTs signaling is provided by exploiting the same OSC used by OMs in time sharing.

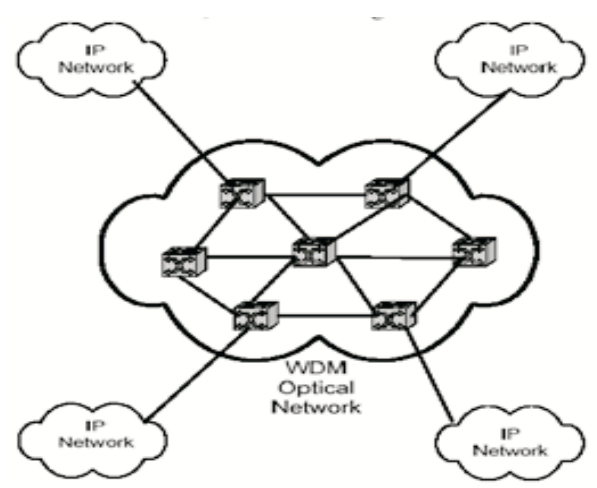

Fig. 3 WDM Network Structure 


\section{A. Network Model}

\section{Features OF MULTI-DOMAIN NETWORK}

A multi-domain network composed of M-connected domains can be represented by a graph $\mathrm{G}=(\mathrm{Di}, \mathrm{E})$ for $\mathrm{i}=1,2, \ldots \mathrm{M}$, where Di and E represent, respectively, the graph of Domain number I and the set of interdomain links (link that connect two border nodes in different domains). Then the domain ' $\mathrm{i}$ ' is denoted on $\mathrm{Di}=(\mathrm{GNi}, \mathrm{INi}, \mathrm{ILi}), 1<=\mathrm{i}<=\mathrm{M}$ where $\mathrm{GNi}$, INi and Ili represent the set of Interior nodes and the set of intradomain links in domain i respectively. An intra-domain link connects nodes of the same domain.

The interior node can view only local network information whereas border nodes can view both local information and global information. The nodes through which a primary lightpath enters domain Di. Domain Ingress Node (DINi) and nodes through which a primary lightpath exits domain Di Domain Engress Nodes (DENi).

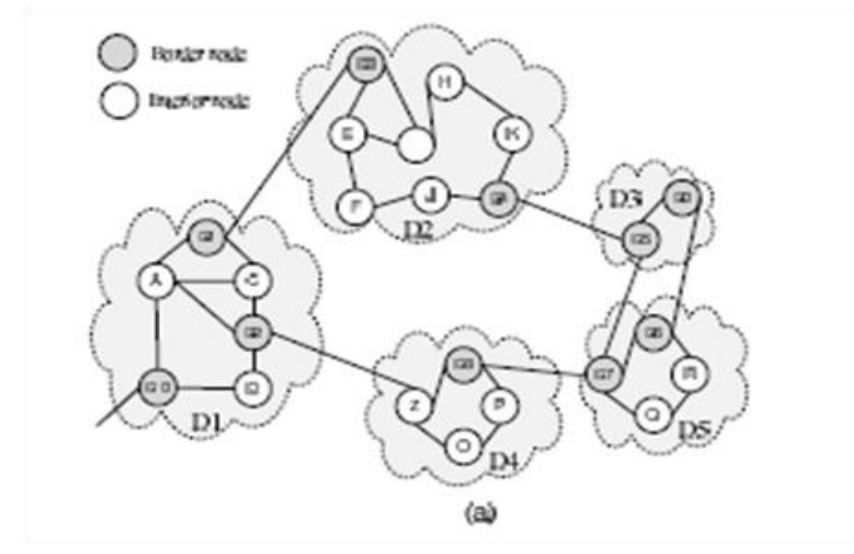

Fig 4. A Multi-domain Network Model

Taking consideration as each connection demand requires one wavelength on all links traversed by its primary lightpath we assume that each node in the network has full wavelength conversion capacity. Our aim is to compare multi-domain survivability schemes so assumption is made for simpler calculation.

\section{SURVIVABILITY IN OPTICAL MULTIDOMAIN WDM NETWORKS}

Methods for ensuring survivablility in WDM network can be classified into two main classes: restoration and protection. Restoration is reactive approach in which the backup lightpath is identified and its resources are reserved at the time of establishing primary lightpath itself. Protection scheme is considered the favorite mechanism for survivability of WDM networks. This is because it generates full recovery whereas restoration comes at the expense of a higher consumption of bandwidth resources.

Protection scheme can be classified according to the type of resources used for protection. In link based protection for each link of a primary lightpath a backup lightpath is identified. Whereas in path based protections are backup lightpath is selected to protect all the links in the primary lightpath.

\section{A. Approaches for survivability issue against Multilink failures.}

a. LBSR Algorithm

This algorithm is proposed to protect failures in multi-domain WDM optical networks with optimum blocking probability and also throughput [1]. Domain to domain routing (DDR) algorithm is used to find the intra-domain subworking path and subbackup path in each single domain to form the inter-domain working path and backup path for each demand. Load balance algorithm is used in order to reduce blocking probability which encourages traffic be uniformly distributed on the links with more free wavelengths [5].

\section{b. ESPP algorithm:}

Enhanced Shared path protection is used while computing backup paths so that more backup resources can be saved. A recovery escalation mechanism is present that is performed when multilink failures affect primary and backup paths simultaneously so that multilink failures can be protected effectively [1].

\section{c. LSPP algorithm:}

Local Segment Protection for multi-domain optical mesh networks is proposed to improve recovery time of ESPP and avoid the notification of failures to all nodes on the primary light path [2]. To protect the end to 
end lightpath, LSPP selects the available path with the minimum number of virtual links hops as the path on virtual topology and map each virtual link of the primary lightpath onto the intra-domain physical topology. Assumption was made that inter-domain links are equipped with one dedicated protection link.

\section{d. P-cycle protection in multi-domain optical networks:}

In p-cycle protection, mainly the inter-domain links are protected. There are three steps [9]. In first step, the set of p-cycle protecting the inter-domain links is computed. The second step determines the p-cycle calculated in previous step that may be like (D1-D2-D3-D1), the border nodes to which the p-cycles are connected (on cycle and straddling links). On-cycle links are (G-A, B-K), straddling links are (D-Z,D-Y). During the last step each internal virtual link calculated in the second step is translated to physical light path. In this the calculated p-cycles bypass the intra-domain links without possibility of protecting them [2].

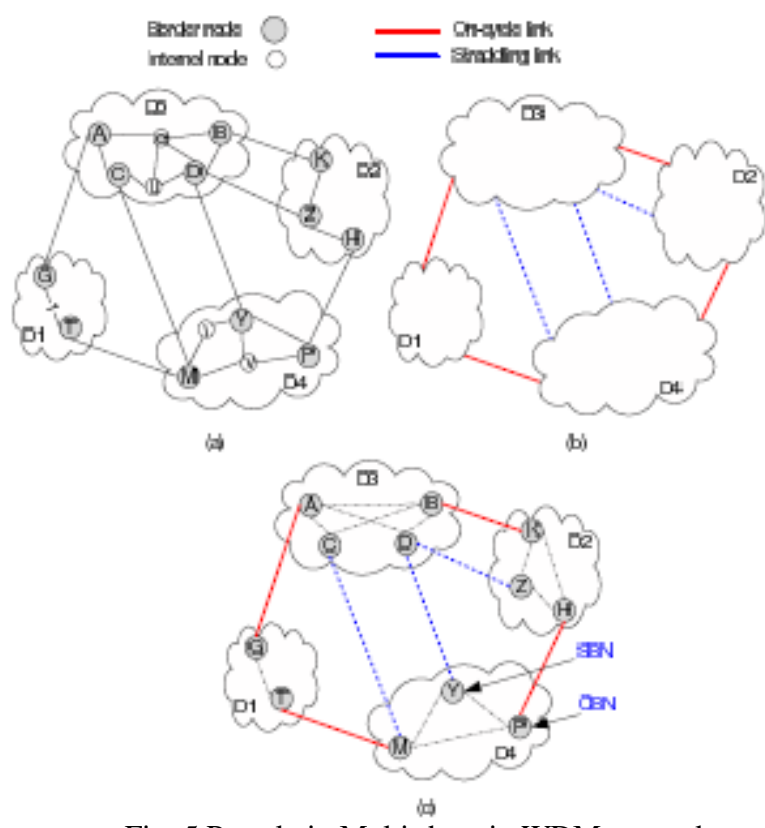

Fig. 5 P-cycle in Multi-domain WDM network

\section{Proposed Algorithm}

With regards of the existing algorithm, in this paper the new approach is introduced. The p-cycle algorithm for protection and the wavelength assignment algorithm along with the Dijkstra's algorithm is combined together to reduce the network layer blocking and the physical layer blocking.

The following shows the new proposed algorithm,

Call request is send from the source.

1. Find all the possible p-cycles.

2. From 1. Find sorted shortest p-cycle paths.

3. In each path look for availability of wavelength.

(i) If there are no wavelengths available it is physical layer blocking and call is blocked.

(ii) If wavelength is available then assign the wavelength for each p-cycles.

4. If link say ' LK' fails,

(i) Go to p-cycles light-path which is not connecting ' LK' link and go to 4.(ii)

(ii) Else select best p-cycle light-path.

5. If there are no p-cycle light-paths, it is network blocking or go to call establishment. 


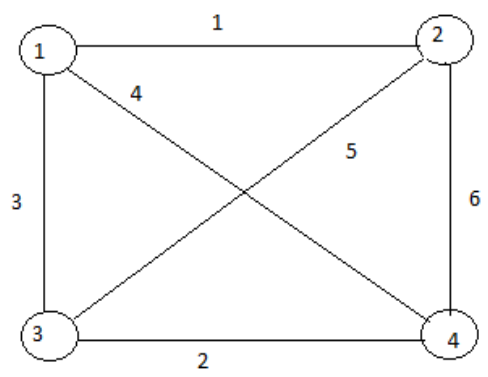

Fig. 6 Four nodal topology showing border nodes of each domain connected by inter-domain links.

\section{CONCLUSION}

In this paper survey on survivability of multi-domain WDM network has been carried out. Different approaches and methods which has been carried out previously are briefly discussed in this paper. The network model, topology and different measures for WDM network have been discussed. P-cycle protection is considered quite remarkable. Dijkstra's algorithm and wavelength assignment has been done so as to find the shortest possible lightpath in the network and to reduce the blocking probability for the continuous flow of signal with optimal resources.

Further we will be converting the virtual topology to the real scenario as accordance to the network structure.

\section{References}

[1] Hamza Drid, Bernard Cousin,Miklos Molnar,Samer Lahoud"A Survey of Survivability in Multi-Domain Optimcal Networks", Campus Uiversitaire de Beaulieu 35042 Rennes CEDEX, France. pp. 1-10, Jan 302010.

[2] A. Nisar and I. Hyder "Routing and Survivability Issues in Optical WDM Networks", TECHNOLOGY FORCES (Techical Forces): Journal of Engineering and Sciences, pp. 27-32, July-December 2009.

[3] Baibaswata Mohapatra, Rajendra K. Nagaria, and Sudrshan Tiwari, " Guarenteed Protection in Survivable WDM Mesh Networks- New ILP Fornulation for Link Protection and Path Protection”, Journal Of Telecommunication And Information Technology, pp. 108-113, April 2011.

[4] Guido Maier, Achille Pattavina, Simone De Patre, Mario Martinelli, “ Optical Network Survivability: Protection Technique in the WDM Layer", Photonic Network Communications, 4:3/4, 251-269,2002.

[5] Kayi Lee, Eytan Modiano, "Cross Layer Survivability In WDM-Based Networks”,NSF grants CNS-0626781 and CNS-0830961 and DTRA grant number HDTRA 1-07-1-0004.,January 2010.

[6] Jeffery Kennington Eli Olinick Augustyn Ortynski and Gheoraghe Spiride, "Robust Solution for the WDM Routing and Provisioning Problem With Uncertain Demands and Single Link Failure Protection”,Technical Report 01-EMIS07, 19 November 2001.

[7] Desalegn Segni Abawollo, "Impairment-aware Survivable Routing and Regenarator Placement in WDM Networks", Master Of Science Thesis,August 312010.

[8] Qi Deng and Galen Sasaki, Ching-Fong Su, "Survivable IP over WDM: An Efficient Mathematical Programming Problem Formulation", Paper From Fujitsu Laboratories Of America,2008.

[9] Mandip Kumar Sandhu and Amit Kumar Garg, "A survey of P-cycle For WDM Networks", urnal Of Information and Operation Management, Issn:0976-7754 and E-Issn:097Jo6-7762,volume3,Issue 1,2012,pp-57-60,December 12,2011.

[10] D.Sheela and C.Chellamuthu, "Protection In Optical Mesh Networks: A Cost Effective Strategy Based On Topological Constraints",Malayasian Journal Of Computer Science, Vol.25(1)pp-38-55,2012. 